\title{
O Uso do Role-Play no Ensino da Técnica de Anamnese e de Habilidades de Comunicação para Estudantes de Medicina
}

\section{The Use of Role-Play in Teaching Anamnesis Technique and Communication Skills for Medical Students}

Carlos Alberto Engelhorn ${ }^{I}$

\section{PALAVRAS-CHAVE}

- Comunicação.

- Medicina.

- Simulação.

Contexto: Comunicação adequada é uma habilidade clínica e, portanto, deve fazer parte dos currículos nas escolas médicas. Várias técnicas podem ser empregadas para ensinar habilidades de comunicação aos estudantes de Medicina. Objetivo: Relatar experiência baseada na utilização da técnica de role-play na aprendizagem e satisfação dos estudantes de Medicina na realização da entrevista médica. Métodos: Estudo transversal com alunos da disciplina de Semiologia em três etapas distintas: entendimento da teoria, aplicação prática na simulação por representação de papéis (role-play) entre os alunos e gravação de vídeo com pacientes internados no hospital.O desempenho dos estudantes no role-play e no vídeo gravado foi avaliado com base em instrumento adaptado do Guia Calgary-Cambridge. Também foi realizada uma pesquisa de satisfação com os estudantes no final do semestre. Resultados: Foram avaliados 30 estudantes, 43\% dos quais não foram capazes de detalhar adequadamente os sintomas durante a simulação, porém na gravação do vídeo não apresentaram esta dificuldade. Em relação ao desempenho na simulação e no vídeo, cinco estudantes (16,6\%) mantiveram o mesmo desempenho; um estudante (3,3\%) apresentou desempenho inferior; e 25 estudantes (83\%) apresentaram melhora do desempenho na entrevista com os pacientes em relação à simulação. A grande maioria (92,5\%) dos estudantes considerou a atividade útil na sua formação. Conclusão: $O$ uso da técnica de role-play no ensino da anamnese apresentou-se como um método de aprendizagem útil e foi bem aceito pelos estudantes de Medicina participantes deste estudo. 


\section{KEY-WORDS}

- Communication.

- Medicine.

- Simulation.
ABSTRACT

Context: Suitable communication is a clinical skill and therefore should be part of the curriculum in medical schools. Several techniques can be used to teach communication skills to medical students. Objective: To report an experience based on the role-play technique in the learning process and the satisfaction of medical students in the application of the medical interview. Methods: A cross-sectional study with students of the discipline of semiology in three distinct stages related to theory comprehension, practical application with role-play simulation among students and taped interviews with patients in the hospital. Student performance evaluation during simulation and video recording was based on an instrument adapted from the Calgary-Cambridge Guide. A student satisfaction survey was also conducted at the end of the semester. Results: Thirty students were evaluated, 43\% were not able to adequately detail the symptoms during the simulation but did not present the same difficulty in the taped video. When comparing the simulation and the taped videos exercise 5 students (16.6\%) showed the same performance, 1 student (3.3\%) displayed a worse performance with the patient interview and 25 students (83\%) improved their performance with patients. The vast majority (92.5\%) of the students considered the activity useful in their medical training. Conclusion: The use of the role-play technique in the teaching process of anamnesis and in the development of communication skills was demonstrated as a useful learning method and was well accepted by the medical students that participated in this study.

Recebido em: 15/11/18

Aceito em: 20/1/19

\section{INTRODUÇÃO}

Estima-se que um médico durante a sua carreira realize aproximadamente 200 mil entrevistas médicas para a solução dos diversos desafios diagnósticos ${ }^{1}$.

Para obter adequadamente as informações durante a entrevista e estabelecer uma boa relação com o paciente, o médico precisa saber falar de maneira clara e eficaz, e, principalmente e o que é mais difícil, saber ouvir².

Em um estudo clássico, Beckman e Franke ${ }^{3}$ demonstraram que os médicos tendem a interromper os pacientes em média 18 segundos após o início da conversa, sendo que somente $23 \%$ dos pacientes tiveram a oportunidade de finalizar os detalhes de sua queixa principal antes de serem interrompidos por seus médicos.

Pacientes preferem entrevistadores que se apresentem, sejam autoconfiantes, simpáticos e amigáveis, de fácil diálogo, sabendo ouvir e responder adequadamente às perguntas ${ }^{4}$. No entanto, o ensino de habilidades de comunicação nem sempre está previsto nos currículos dos cursos de Medicina.

Entre várias técnicas que podem ser utilizadas para ensinar habilidades de comunicação aos estudantes de Medicina, o role-play pode facilitar o desenvolvimento da comunicação adequada com pacientes e familiares, incluindo aspectos afetivos e emocionais ${ }^{5,6}$.
A disciplina de Semiologia 1 da Pontifícia Universidade Católica do Paraná estudada nesta pesquisa é composta por aulas teóricas e práticas à beira do leito e de habilidades de comunicação nas quais são abordados aspectos teóricos e práticos da relação médico-paciente e da técnica de anamnese. No módulo de habilidades de comunicação, há o treinamento prático da técnica de anamnese e de habilidades de comunicação com uso da simulação clínica por representação de papéis (role-play).

Trata-se de um treinamento em pequenos grupos diante dos colegas com a mediação do professor, cuja finalidade é adquirir o domínio da técnica de anamnese e o desenvolvimento de habilidades de comunicação

O objetivo deste trabalho foi relatar uma experiência baseada na utilização da técnica de role-play na aprendizagem e satisfação de estudantes de Medicina na realização da entrevista médica.

\section{MÉTODOS}

Foi realizado um estudo transversal com alunos do primeiro ano de um curso de Medicina matriculados na disciplina de Semiologia 1 em três etapas distintas: discussão e entendimento da teoria, aplicação prática da teoria por simulação (role-play) entre os alunos e gravação de vídeo com paciente 
no hospital-escola. O estudo foi aprovado pelo Comitê de Ética em Pesquisa da referida instituição sob o protocolo $n^{\circ}$ 65161317.0.0000.0020.

Nas primeiras semanas da disciplina, foram discutidos os aspectos teóricos da anamnese, incluindo tipos de perguntas e escuta e a técnica de entrevista médica. Nesta etapa, o professor auxiliou os estudantes no entendimento teórico pela exemplificação prática (no papel de paciente) de situações de como fazer as perguntas adequadas, saber ouvir, incentivar o paciente a falar, estabelecer um vínculo psicológico favorável, ler e interpretar as atitudes corporais de uma linguagem não verbal.

Após a discussão dos aspectos teóricos da técnica de anamnese, na segunda etapa houve o sorteio de doenças prevalentes na população brasileira para cada um dos estudantes que pesquisaram as características da doença, sobretudo os sintomas que a determinam, criando um personagem adequado a faixa etária e sexo.

Em cada aula houve o sorteio de duplas para a simulação. O estudante no papel de paciente apresentou os sintomas da doença previamente preparada sem o conhecimento dos colegas, e o estudante no papel de médico realizou a anamnese, utilizando as habilidades de comunicação e a técnica de entrevista em função dos sintomas apresentados pelo personagem paciente.

Durante as simulações, os demais colegas acompanharam atentamente o desenrolar das anamneses e no final discutiram em grupo a performance dos dois colegas, sobretudo do médico, com feedback do professor.

Na última etapa da disciplina, foi solicitado que cada estudante gravasse um vídeo com pacientes internados no hospital, os quais assinaram o Termo de Consentimento Livre e Esclarecido autorizando a gravação da entrevista. O desempenho nesta etapa foi comparado com o da simulação.

A avaliação de desempenho dos estudantes na simulação e no vídeo gravado foi baseada em um instrumento adaptado ${ }^{7}$ do Guia Calgary-Cambridge que avaliou as etapas da entrevista (identificação, queixa principal, história mórbida atual, perfil psicossocial, história mórbida pregressa, história mórbida familiar, condições e hábitos de vida e revisão de sistemas) e o aspecto comportamental (ouvir com interesse e respeito, evitar interromper o paciente, usar linguagem adequada, esclarecer termos, linguagem não verbal adequada e anotar adequadamente as informações).

No final da disciplina, foi solicitado que os estudantes anonimamente respondessem a um questionário sobre a utilidade da simulação na aprendizagem da técnica de anamnese, as dificuldades encontradas e a segurança em realizar a entrevista nos futuros períodos e atividades do curso.

\section{RESULTADOS}

Foram avaliados 30 estudantes, 7 homens e 23 mulheres, devidamente matriculados e que cursaram o módulo de habilidades de comunicação da disciplina durante todo o semestre.

Com referência à avaliação dos estudantes na fase de simulação (role-play) considerando as etapas da técnica de anamnese, um estudante $(3,3 \%)$ apresentou dificuldades na obtenção da identificação completa do paciente e nenhum estudante apresentou limitações na obtenção da queixa principal.

Durante a obtenção da história mórbida atual, somente um estudante $(3,3 \%)$ não foi capaz de identificar todos os sintomas preparados pelo paciente de acordo com a doença sorteada. Contudo, 43\% dos estudantes não foram capazes de detalhar adequadamente todos os sintomas dos pacientes, sendo que $40 \%$ não utilizaram perguntas abertas e $13 \%$ usaram perguntas compostas ou dirigidas. Além disso, dois estudantes $(6,6 \%)$ não realizaram sumário ao término da obtenção da história mórbida atual.

Em relação ao perfil psicossocial, história mórbida pregressa, história mórbida familiar e costumes de hábitos de vida, $10 \%, 23 \%$, $13 \%$ e $10 \%$, respectivamente, realizaram estas etapas de maneira incompleta.

Considerando as habilidades de comunicação durante as simulações, todos os estudantes ouviram com interesse e respeito os pacientes simulados, sem interrupções desnecessárias. Um estudante (3,3\%) utilizou linguagem técnica inadequada ao paciente em questão, um (3,3\%) apresentou dificuldades para anotar as informações durante a entrevista e $11(36 \%)$ demonstraram sinais não verbais de nervosismo e insegurança durante a anamnese.

Quanto à avaliação dos estudantes na gravação dos vídeos com os pacientes considerando as etapas da técnica de anamnese, nenhum estudante apresentou dificuldades na obtenção da identificação do paciente, na obtenção da queixa principal, identificação dos sintomas e detalhamento dos sintomas na história mórbida atual. Dois estudantes $(6,6 \%)$ não realizaram o sumário ao término da história mórbida atual. Todos os estudantes fizeram perguntas abertas, mas três estudantes $(10 \%)$ utilizaram perguntas dirigidas ou compostas.

Em relação a perfil psicossocial, história mórbida pregressa e história mórbida familiar, nenhum estudante apresentou dificuldades. Na investigação dos costumes e hábitos de vida, seis estudantes (20\%) investigaram os hábitos de vida de maneira incompleta.

Considerando as habilidades de comunicação durante as gravações, todos os estudantes ouviram com interesse e respeito os pacientes, evitando interrompê-los, com uso de linguagem adequada. Um estudante (3,3\%) demonstrou si- 
nais não verbais de nervosismo e insegurança durante a entrevista.

Em relação ao desempenho dos estudantes na simulação e na gravação com os pacientes no hospital, cinco estudantes $(16,6 \%)$ mantiveram o mesmo desempenho; um estudante $(3,3 \%)$ apresentou desempenho discretamente inferior com um paciente prolixo e confuso; e 25 estudantes (83\%) apresentaram melhora do desempenho na entrevista com os pacientes em relação à simulação.

Vinte e sete estudantes responderam ao questionário no final do semestre letivo, sendo que $81 \%$ se sentiram seguros para realizar a entrevista e cinco estudantes (19\%) relataram ainda um pouco de insegurança por falta de experiência e conhecimento médico.

Em relação aos problemas enfrentados durante as anamneses, além da dificuldade inicial de memorizar as etapas da entrevista, $40 \%$ dos estudantes referiram dificuldades no detalhamento de múltiplos sintomas por falta de conhecimento das doenças e dois estudantes $(7,4 \%)$ sentiram-se desconfortáveis na exploração da história sexual dos pacientes. A organização da anamnese e o modo adequado de anotar as informações também foram referidos como dificuldades.

Questionados sobre a utilidade da simulação na aprendizagem da anamnese, 92,5\%dos estudantes consideraram a atividade útil na sua formação e ressaltaram a possibilidade de errar em um ambiente sem o paciente real, vivenciar previamente situações difíceis e inusitadas, ganhar confiança antes de conversar com o paciente real e aprender com os erros dos colegas. Somente um estudante referiu desconforto no papel de paciente e um estudante considerou a simulação artificial.

\section{DISCUSSÃO}

A competência clínica dos médicos depende da integração de diversos aspectos: conhecimento médico, habilidades de comunicação, exame físico, raciocínio clínico e capacidade de resolver problemas.

Existem elementos essenciais de comunicação e de relacionamento na consulta médica cujo objetivo primordial é a construção de uma boa relação médico-paciente. Quanto à comunicação, é necessário iniciar de maneira adequada a conversa, obter as informações relevantes, entender a perspectiva do paciente, dividir informações, definir de comum acordo o plano de ação e encerrar a sessão sem dúvidas para o paciente ${ }^{8,9}$.

Existem três habilidades de comunicação que devem ser abordadas no ensino dos estudantes: conteúdo (o que é comunicado, a informação obtida e transmitida); processo (como a comunicação verbal e não verbal ocorre) e percepção (pensamentos e sentimentos) $)^{1}$.
Comunicação adequada é uma habilidade clínica e, portanto, deve fazer parte dos currículos e ser ensinada nas escolas médicas. Diferentes métodos podem ser empregados no ensino de habilidades de comunicação, como a observação de entrevistas com pacientes, vídeos, uso de atores e pacientes simulados e role-play.

O uso do role-play na educação médica está baseado nas assertivas definidas por Knowles et al. ${ }^{10}$ sobre a educação de adultos, nas quais os estudantes são independentes e autodirecionados; têm experiência que pode ser fonte de aprendizagem; valorizam ensinamentos que têm relação com suas necessidades do dia a dia; são mais interessados na resolução imediata de problemas, em vez de assuntos subjetivos; são mais motivados a aprender por interesses pessoais;e necessitam de feedback construtivo.

O role-play é uma técnica de ensino na qual os participantes assumem determinado papel e interagem com outras pessoas em um cenário específico, tentando agir como o personagem agiria na situação que imita a vida real e que pode ser aplicada em diversos aspectos da comunicação médico-paciente. ${ }^{11,12}$

Essa técnica pode ser utilizada com pacientes simulados, atores, estudantes veteranos ou mesmo colegas de classe e está baseada numa série de etapas: (a) preparação da atividade pelo facilitador; (b) criação de um ambiente seguro e favorável à aprendizagem; (c) escolha e uso do cenário a ser simulado; (d) condução da sessão; (e) feedback ${ }^{13}$.

Cada etapa deve ser planejada e conduzida pelo facilitador a fim de que num ambiente adequado os estudantes possam efetivamente fazer parte do processo seja na função de personagens, seja como observadores no grupo.

Em nosso estudo, optamos por utilizar os estudantes colegas de classe nos papéis de médicos e pacientes. Os alunos foram previamente expostos aos aspectos teóricos da anamnese e da relação médico-paciente e puderam observar e participar de simulações realizadas pelo facilitador no papel de paciente, demonstrando como utilizar perguntas adequadas e escuta eficaz.

Essa etapa inicial, além de facilitar o entendimento teórico e o papel do médico e paciente na simulação, foi responsável por "quebrar o gelo" entre os integrantes do grupo e criar um ambiente descontraído.

Existem várias opções na utilização do role-play no treinamento de habilidades de acordo com o objetivo a ser atingido. Se o objetivo for treinamento de como dar más notícias, pode ser usada a técnica chamada Spikes (Setting, Perception, Invitation, Knowledge, Emotions, Strategy, andSummary), na qual o facilitador assume o papel de médico, e os estudantes, de pacientes e familiares, ou os estudantes assumem tanto o papel 
de médicos como de pacientes e familiares ${ }^{14}$. Outra possibilidade é a escolha do estudante como paciente para que ele possa entender a perspectiva do doente na relação médico-paciente.

Como o objetivo do nosso estudo era o conhecimento e entendimento da técnica de anamnese, os cenários escolhidos pelo facilitador e os personagens criados pelos estudantes foram baseados nas doenças mais prevalentes na população brasileira, tendo-se optado pelo modelo no qual os estudantes assumiam tanto o papel de médico como o de paciente, de tal forma que pudessem vivenciar os dois aspectos da relação médico-paciente.

Conforme sugerido por Loyd e Bor ${ }^{15}$, durante a sessão de role-play não houve interferência do facilitador. Os estudantes eram encorajados a realizar todo o processo da entrevista médica e no final eram questionados como se sentiram durante o processo e quais foram suas dificuldades e limitações tanto no papel de médico como de paciente. Após uma autoavaliação e reflexão, o estudante médico recebia o feedback de sua performance dos demais integrantes do grupo e, finalmente,do professor.

Essa avaliação pelos pares mostrou-se factível e útil, conforme demonstrado também por Sommeret al. ${ }^{16}$ em um estudo inspirado no modelo Calgary-Cambridge centrado em pacientes e estudantes como foco do processo de aprendizagem.

O feedback do professor foi focado na performance dos alunos tanto na aplicação da técnica de anamnese quanto nas habilidades de comunicação e também pela utilização do guia modificado.

O Guia Calgary-Cambridge, proposto por Kurtzet al. ${ }^{1} \mathrm{e}$ Silvermanet $a l,{ }^{17}$ é composto por 55 itens e define as habilidades individuais que o estudante de Medicina deve desenvolver para a obtenção da anamnese, incluindo:(a) iniciar a sessão para estabelecer o contato inicial com o paciente e descobrir o motivo da consulta; (b) obter as informações, incluindo a exploração dos problemas, o entendimento da perspectiva do paciente e uso de entrevista estruturada; (c) fornecer informações ao doente, incluindo tomada de decisão;(d) fechar a sessão com sumário e próximos passos.

Devido à inexperiência clínica dos estudantes do segundo período do curso que fizeram parte do nosso estudo, optamos por uma versão mais concisa do guia, com 20 itens focados na técnica de anamnese e nas habilidades de comunicação, excluindo os itens relacionados ao fornecimento de informações médicas e tomada de decisão.

Segundo a avaliação realizada pelo instrumento adaptado durante o role-play, $43 \%$ dos estudantes não foram capazes de detalhar adequadamente todos os sintomas dos pacientes, demonstrando que a falta de conhecimento médico prévio pode ser um fator limitador no início do treinamento. Contudo, no final do semestre, após a exposição aos sintomas das doenças mais prevalentes em nossa população, durante a gravação do vídeo com os pacientes internados no hospital, os estudantes não demonstraram a mesma dificuldade na identificação e detalhamento dos sintomas na história mórbida atual.

Considerando as habilidades de comunicação durante o role-play, apesar dos sinais não verbais de nervosismo e insegurança durante a anamnese, todos os estudantes ouviram com interesse e respeito os pacientes simulados, sem interrupções desnecessárias e com linguagem adequada.

O mesmo foi observado durante as gravações dos vídeos, sendo que somente um estudante demonstrou sinais não verbais de nervosismo e insegurança durante a entrevista, porém sem interrupções desnecessárias na conversa.

Muitas vezes, a interrupção do paciente pode ser necessária, mas há uma linha tênue entre despertar no doente a sensação de respeito ou desrespeito, e por isso é necessário que o interlocutor eventualmente se desculpe pela interrupção, explicando o motivo da interrupção e enfatizando o ponto a ser explorado ${ }^{18}$.

Foi observado um desempenho melhor na gravação do vídeo, demonstrando que, após a aquisição de conhecimento sobre os sintomas das doenças, o treinamento pessoal com feedback construtivo pelo grupo e pelo facilitador e a observação de erros dos colegas, há uma tendência de melhora da performance dos estudantes.O uso do vídeo apresenta várias vantagens, tais como ser centrado no estudante, evitar erros de interpretação e permitir feedback mais específico e descritivo ${ }^{1}$.

Apesar das dificuldades de memorizar as etapas da anamnese, e de detalhar os sintomas apresentados pelos pacientes, a maioria dos estudantes sentiu-se segura para realizar as entrevistas e considerou a atividade útil em sua formação, ressaltando a possibilidade de errar em um ambiente sem o paciente real, vivenciar previamente situações difíceis e inusitadas, ganhar confiança antes de conversar com o paciente real e aprender com os erros dos colegas.

A percepção dos nossos estudantes de que o laboratório de habilidades de comunicação é útil na formação é corroborada por dois estudos indianos, conduzidos por Tanwaniet al. ${ }^{19} \mathrm{e}$ Choudhary e Gupta ${ }^{20}$.

O estudo de Tanwanietal. ${ }^{19}$ utilizou uma metodologia semelhante à nossa, com exposição teórica inicial seguida de prática com pacientes simulados em vez de role-play. Assim como em nosso estudo (92,5\%), a grande maioria dos 60 estudantes indianos participantes $(96,4 \%)$ considerou que a simulação melhorou suas habilidades de comunicação.

$\mathrm{O}$ estudo de Choudhary e Gupta ${ }^{20}$ foi realizado com estudantes de Medicina do último ano do curso, num curso de curta duração, utilizando role-play e pacientes simulados. Os 
estudantes foram avaliados pelo Guia Calgary-Cambridge e avaliaram o curso com questionários pré- e pós-teste. Também a maioria (88\%) dos estudantes considerou o treinamento válido e $90 \%$ apresentaram melhora na comunicação ${ }^{20}$.

Em conclusão, o uso da técnica de role-play no ensino da anamnese e no desenvolvimento de habilidades de comunicação apresentou-se como um método de aprendizagem útil e foi bem aceito pelos estudantes de Medicina participantes deste estudo.

\section{REFERÊNCIAS}

1. Kurtz S, Silverman J, Draper J. Teaching and learning communication skills in Medicine. Radcliffe Medical Press, Abingdon, Oxon,UK, 1998

2. Lown B. A arte perdida de curar. São Paulo: JSN,1997.

3. Beckman HB, Frankel RM. The effect of physician behavior on the collection of data. Ann Intern Med 1984;101(5):692-696.

4. Maguire P, Fairbain S, Fletcher C. Consultation skills of young doctors: benefits of undergraduate feedback training in interviews in Stewart M, Roter D. Communicating with medical patient. Sage Publications, Califórnia, 1989

5. Billings DM. Role-play revisited. J Contin Educ Nurs 2012;43:201Y202

6. Dickson DA, Maxwell M, Saunders C. Using role-play with physiotherapy students. Physiotherapy 1991;77:145-153

7. Mognon F. Avaliação do desenvolvimento das habilidades de comunicação no programa de aprendizagem Estágio em semiologia e práticas hospitalares I, baseada em mapas cognitivos. Dissertação de Mestrado. Pontifícia Universidade Católica do Paraná, Curitiba, 2007.xii, 194 f.: il.

8. Makoul G. Essential elements of communication in medical encounters: the Kalamazoo consensus statement. Acad Med 2001;76 (4) 390- 393

9. Mauksch LB, Dugdale DC, Dodson S et al. Relationship, Communication, and Efficiency in the Medical Encounter Creating a Clinical Model from a Literature Review. Arch Intern Med. 2008;168(13):1387-1395

10. Knowles MS, Holton EF, Swanson RA. The Adult Learner: The Definitive Classic in Adult Education and Human Resource Development. 6th ed. Elsevier; Burlington, MA; 2005

11. Simpson MA. How to use role-play in medical teaching. Medical Teaching 1985; 7(1): 75-82

12. Lavanya SH, Kalpana L, Veena RM, Kumar VDB. Role-play as an educational tool in medication communication skills: Students' perspectives. Indian J Pharmacol. 2016 Oct; 48(Suppl 1): S33-S36.
13. BaileWF,Blatner A. Teaching Communication Skills Using Action Methods to Enhance Role-play in Problem-based Learning. Sim Healthcare 2014, 9:220-227

14. Baile WF, Buckman R, Lenzi R, Glober G, Beale EA, Kudelka AP. SPIKES-A six-step protocol for delivering bad news: application to the patient with cancer. Oncologist 2000;5:302-311

15. Lloyd M, Bor R. Communication Skills for Medicine. Churchill Livingstone, 2009

16. Sommer J, Lanier C, Perron NJ, Nendaz M, Clavet D, Audétat MC. A teaching skills assessment tool inspired by the Calgary-Cambridge model and the patient-centered approach.Patient Educ Couns 2016;99(4):600-9.

17. Silverman JD, Kurtz SM, Draper J. Skills for Communicating with Patients. Radcliffe Medical Press (Oxford), 1998

18. Mauksch LB. Questioning a Taboo Physicians' Interruptions during Interactions with Patients. JAMA 2017;143(17): 1021-2

19. Tanwani R, Chandki R, Joshi A, Arora KV, Nyati P, Sutay S. Perception and Attitude of Medical Students Towards Communication Skills Lab and Teaching Module. Journal of Clinical and Diagnostic Research 2017; 11(6): JC12-JC14.

20. Choudhary A e Gupta V. Teaching communications skills to medical students: Introducing the fine art of medical practice. Int J Appl Basic Med Res. 2015 Aug; 5(Suppl 1): S41-S44.

\section{CONTIBUIÇÃO DO AUTOR}

Concepção e desenho do estudo; Análise e interpretação dos dados; Coleta de dados; Redação do artigo; Revisão crítica do texto; Aprovação final do artigo; Responsabilidade geral pelo estudo: Carlos Alberto Engelhorn

Carlos Alberto Engelhorn

Rua José Casagrande, 1310

CEP 80820-590 - Curitiba - PR

Mestre em Educação pela PUCPR, Doutor em Cirurgia Vascular pela UNIFESP e Professor Titular de Angiologia da PUCPR carlos.engelhorn@pucpr.br

Conflito de interesse: nenhum

\section{AGRADECIMENTOS}

O autor agradece o suporte financeiro do FINEP para a implementação da prática e realização da pesquisa. 